\title{
Quantum light for biomedical imaging
}

\begin{tabular}{|c|c|c|}
\hline $\begin{array}{l}\text { Normally, when we photograph } \\
\text { an object, we rely on visible } \\
\text { light from the sun that is } \\
\text { captured directly by the } \\
\text { camera sensor. When it comes } \\
\text { to looking at cells and tissue } \\
\text { samples though, it is often } \\
\text { better to use infrared light. } \\
\text { Inna Kviatkovsky and Dr Sven } \\
\text { Ramelow at the Humboldt } \\
\text { University Berlin, together } \\
\text { with their collaborators } \\
\text { at Max Delbruck Center } \\
\text { for Molecular Medicine } \\
\text { in Berlin, are developing } \\
\text { new quantum imaging } \\
\text { mid-infrared microscopy } \\
\text { techniques to overcome some } \\
\text { of the challenges associated } \\
\text { with working with these } \\
\text { wavelengths of light. }\end{array}$ & $\begin{array}{l}\text { e see objects when the light } \\
\text { they reflect reaches our } \\
\text { eyes and starts a cascade of } \\
\text { complex chemical processes. To trigger } \\
\text { these processes, we have two main } \\
\text { types of receptor in our eye that help } \\
\text { us to see - cones and rods. Rods are } \\
\text { very sensitive to light levels but cannot } \\
\text { process any information on colours, } \\
\text { whereas cones have different responses } \\
\text { to different wavelengths of light and are } \\
\text { what allow us to see in colour. } \\
\text { Whether it is seeing the outline of an } \\
\text { object or working out its colour patterns, } \\
\text { our vision is completely dependent on } \\
\text { the information contained in the light } \\
\text { reflected by the object. We often think } \\
\text { of light as being made of photons, small } \\
\text { packets of light energy that we can } \\
\text { convert to the chemical information we } \\
\text { need to see. For example, an object of } \\
\text { a certain colour will absorb photons of } \\
\text { a certain energy. We see the colour of } \\
\text { an object because, during the reflection } \\
\text { process, some photons will go 'missing' } \\
\text { as they are absorbed by the object. The } \\
\text { colour we see is just the } \\
\text { reflected photons that } \\
\text { make it to our eye. }\end{array}$ & $\begin{array}{l}\text { image is in focus and can even help } \\
\text { maximise the amount of light that } \\
\text { arrives at the sensor when taking } \\
\text { photographs in darker environmental } \\
\text { conditions. } \\
\text { While a microscope might not look } \\
\text { much like a camera, it shares many of } \\
\text { the same components and may even } \\
\text { use exactly the same type of sensor } \\
\text { as a standard camera. However, there } \\
\text { are several technical limitations on } \\
\text { using these sensors for the infrared } \\
\text { wavelengths of light that are often useful } \\
\text { for looking at tissue samples. Rather } \\
\text { than simply designing better sensors, } \\
\text { Inna Kuiatkovsky and Dr Sven Ramelow } \\
\text { at the Humboldt University Berlin, and } \\
\text { their collaborators at Max Delbruck } \\
\text { Center for Molecular Medicine in Berlin, } \\
\text { have completely circumvented the issues } \\
\text { with existing sensor technologies by } \\
\text { demonstrating a new way of using the } \\
\text { quantum properties of light to perform } \\
\text { microscopy in the mid-infrared. } \\
\text { SENsOR LIMITATIONS } \\
\text { There are two main types of } \\
\text { image sensor used in cameras: } \\
\text { charged-coupled devices (CCD) } \\
\text { and complementary metal-oxide- } \\
\text { semiconductor (CMOS) sensors. } \\
\text { They both convert the incident light } \\
\text { into electrical signals but process the } \\
\text { generated charge in slightly different } \\
\text { wayss. The sensors are made of silicon } \\
\text { that, when they absorb light, release } \\
\text { electrons that move to generate a } \\
\text { current. This current is what provides an } \\
\text { electrical signal that can be converted } \\
\text { to a digital signal containing all the } \\
\text { information about the image that the } \\
\text { sensor 'saw'. }\end{array}$ \\
\hline
\end{tabular}

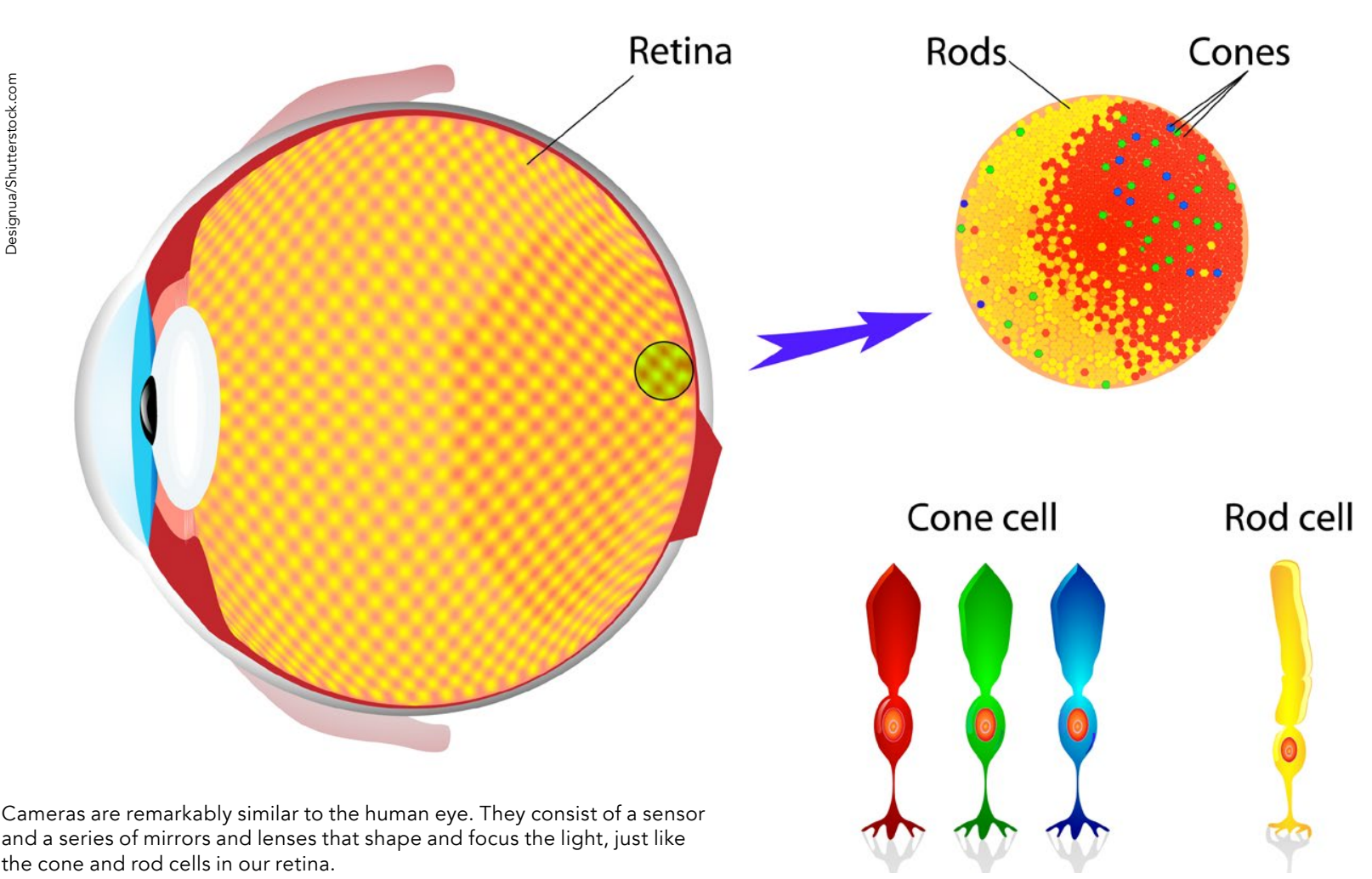

silicon has proved a very popular material as it works reasonably well with a large number of incident wavelengths or photon energies. While it might seem strange to take photographs with wavelengths of light invisible to the human, for biological systems, the most information-rich photographs are often those taken and detected with mid-infrared light. Here, the biological molecules have many unique signatures in their absorption that act as a 'fingerprint
unique identification.

The problem is that it can be difficult to find good ways to illuminate samples with large amounts of mid-infrared light. Many mid-infrared sensors become very noisy and perform poorly in this range of wavelengths. This is why Kviatkovsky and Dr Ramelow tried to find a way of using photons to take photographs using standard mid-infrared sources and sensors in an entirely different way.

\section{QUANTUM LIGHT}

The team's new approach involves shining visible photons from a laser into a crystal. Certanc crystal materials with specic nonla ar properties can be of lower energy. To build their quantum imaging
microscope, Kviatkovsky and Dr Ramelow first focused a standard generate a photon pair. The photons pass through the crystal two times. On each of those trips, there is a possibility that a photon pair will be generated, either on the first or the second trip. These photon pairs are indistinguishable from each other; therefore, there is no way to tell if the second pass through the crystal.

By engineering the crystal, it is possib to generate photons of different photon and a shorter wavelength. visible one. Once the photon pair was generated from the crystal, the team passed the mid-infrared photon through the sample of interest but not the visible one. They were then passed back through the crystal, and the visib photon sent to a CMOS detector.

What this process creates is a non-

The most information-rich photographs are often those taken and detected with mid-infrared light.

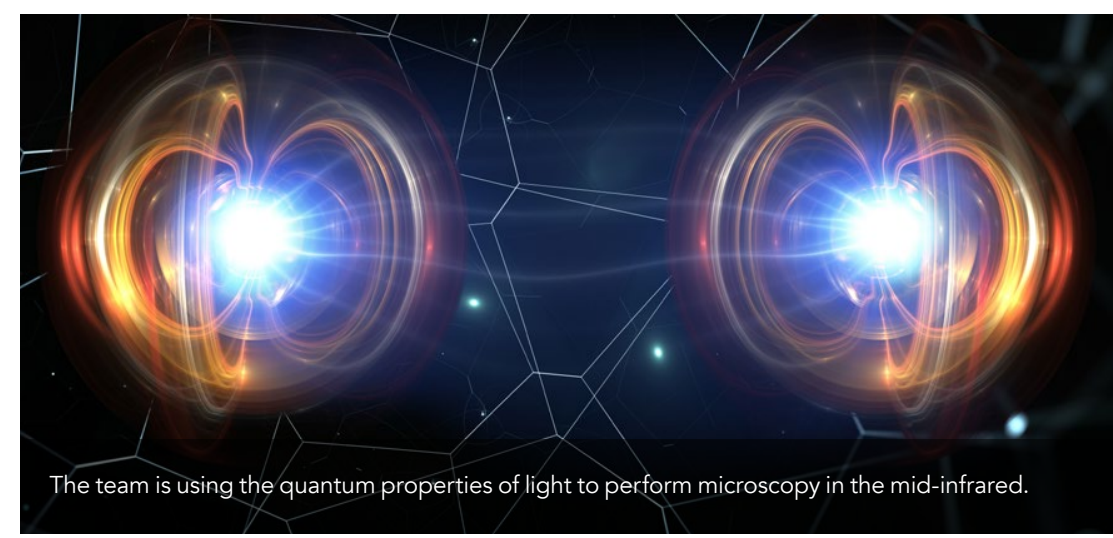
laser into a nonlinear crystal to 


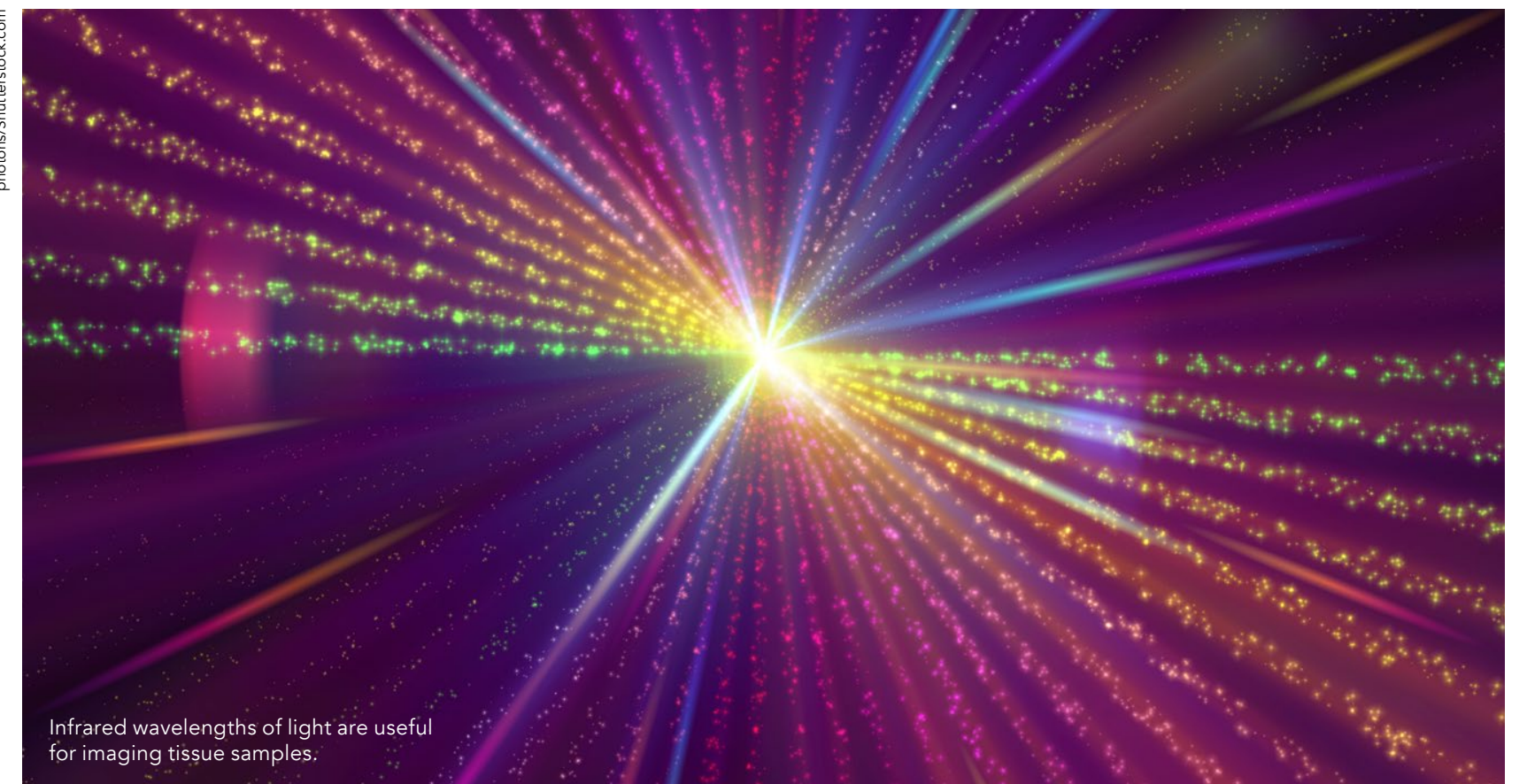

between the two different possible the probability that the photon pair was generated on the first pass through the crystal, but also the possibility that the pair was not generated on the first pass through but instead on the second pass through the crystal. If the mid-infrared photon changes in any way after interacting with the sample, this causes interference-effects which also affect the visible photon. Although object, the information on the object's the undetected infrared photon.

This set-up is a way of detecting the unique fingerprint information that can only be retrieved in the infrared region without ever needing to detect the infrared photon. CCD and CMOS sensors, particularly in low-light conditions, are much more effective at detecting visible photons and Ramelow have found a way to use the

The visible photon never sees the object, the information on the object's composition is transferred to it from the undetected infrared photon.

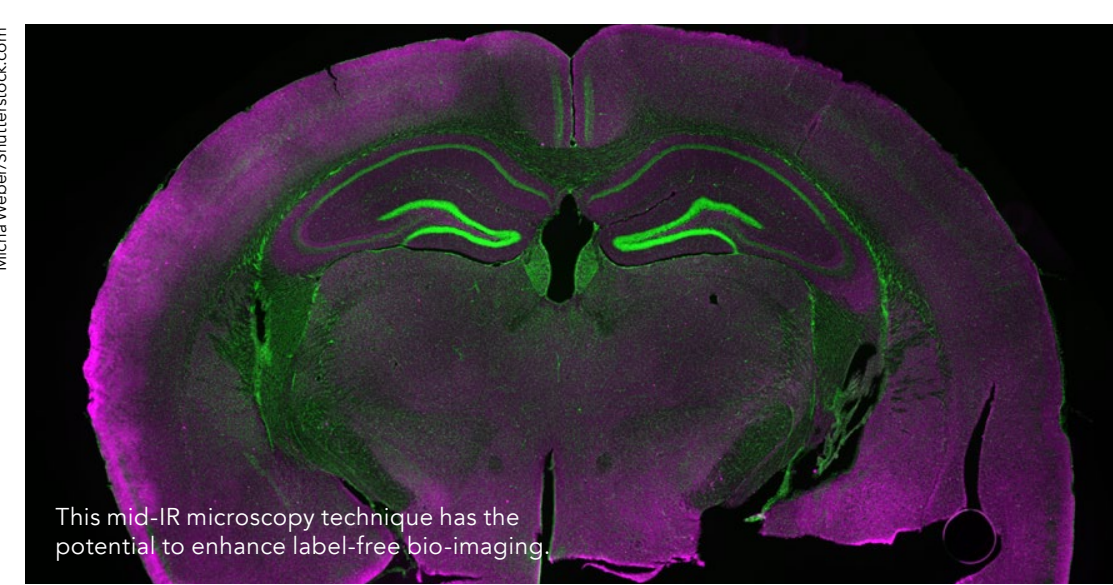

powers of quantum light to improve how mid-infrared microscopy work is done.

\section{SEEING CLEARLY}

While it might seem far more complex than directly measuring the absorption of mid-infrared light by a sample, one of the key advantages of the imaging scheme proposed by Kviatkovsky and Ramelow is that it does not rely on expensive broadband mid-infrared ligh sources and can use readily available visible light detectors which have much etter signal to noise ratios than their

The team have already been able to successfully take images of tissue samples from a mouse heart with their new set up. The measured samples were also unstained and did not require he addition of any tags or biomarkers a common practice in microscopy to try and enhance the strength of the signals that can be detected from the sample.

Another advantage of this new approach is that it is also highly flexible in the colours of light that can be used for it - opening the possibilities to perform to have 'colour vision' when maging samples. This dramatically enhances the amount of information that can be recovered on both the shape and spectral characteristics of a sample, making for an even $\mathrm{m}$

\section{Behind the Research} a

Inna Kviatkovsky

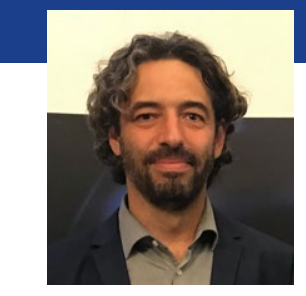

Dr Sven

E: innakv@physik.hu-berlin.de E: sven.ramelow@physik.hu-berlin.de T: +4930 2093 7882/7799 W: https://www.physics.hu-berlin.de/en/niqo

\section{Research Objectives}

The researchers developed a mid-IR microscopy technique with a potential wide range of applications in label-free bio-imaging.

\section{Detail}

Bio

Inna Kviatkovsky studied in Israel (M.Sc. in Biomedical Engineering) before starting her PhD in Berlin, Germany.

Sven Ramelow studied Physics in Germany. After his PhD in Vienna with and Columbia University, USA, before

\section{References}

(42., Bicroscopy with undetected photons in the mid-infrared. Science Advances, 6(42), 1-7. Available at: https://advances.sciencemag.org/content/6/42/eabd0264

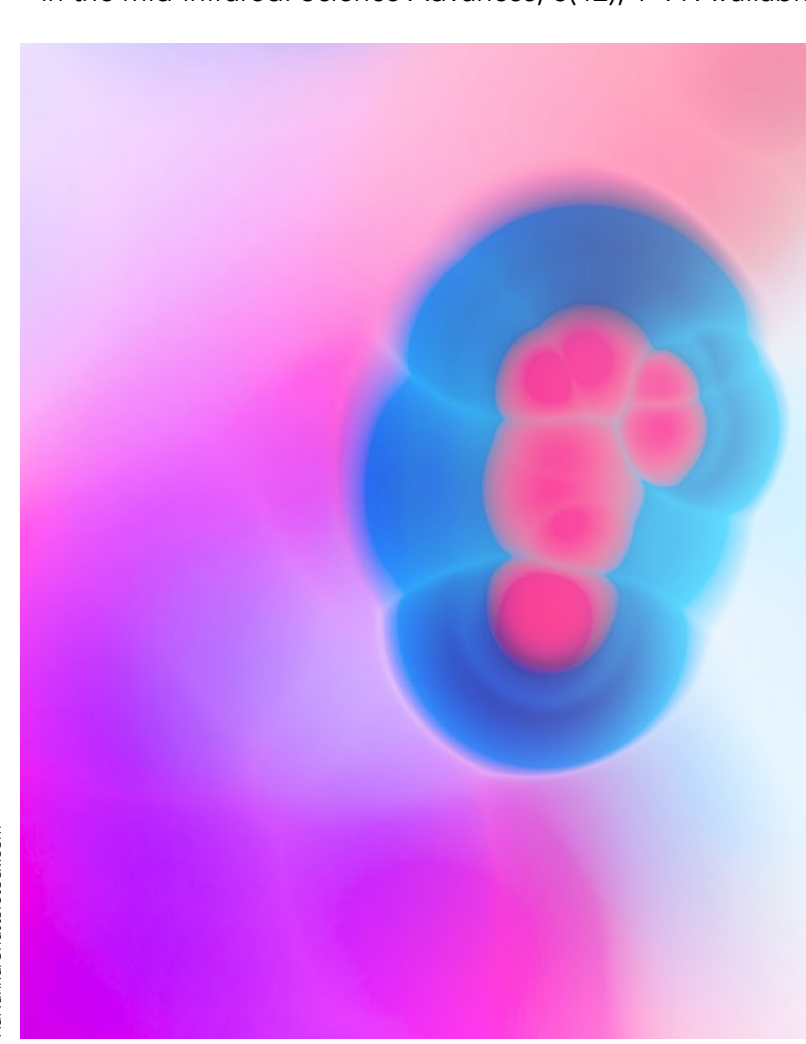

A. Zeilinger, he did research at Cornell Berlin, Germany.

Collaborators Wilck Lab, Max Delbruck Center for Germany

Müller/Dechend Lab, Max Delbruck

Center for Molecular Medicine
(MDC), Berlin, Germany

Funding

The work was funded by Deutsche Forschungsgemeinschaft (RA 2842/1-1) 